\title{
The $\phi$-maze: A versatile automated T-maze for learning and memory experiments in the rat
}

\author{
W. JEFFREY WILSON \\ Indiana University-Purdue University, Fort Wayne, Indiana
}

\begin{abstract}
The $\phi$-maze is a novel implementation of the T-maze that can be used for various behavioral experiments. It consists of a start/goalbox from which a central alley leads to a choice point. Left and right arms return to the start/goalbox, where reinforcers are delivered. A holding chamber at the choice point can be used for delayed (non)matching-to-sample experiments. Lamps mounted under the translucent acrylic floor serve as visual stimuli. The design incorporates reliable and inexpensive automated guillotine doors and a modification of a commercially available pellet dispenser that allows the automated dispensing of Froot Loop reinforcers. The $\phi$-maze has been used successfully in experiments involving spatial and visual alternation, visual discrimination, and delayed matching-to-sample.
\end{abstract}

The history of experimental psychology is replete with experiments involving the T-maze, the Lashley jumping stand, and other apparati that measure discrimination behavior in the rat by means of responses other than leverpresses. These classic apparati share the drawback of requiring the constant involvement of the experimenter throughout each experimental session, notably to return the rat to the starting position so that the next trial can begin. The handling of the subject by the experimenter makes automation of experiments difficult if not impossible. It is perhaps for this reason that the use of the T-maze has been decreasing. An examination of PsycLIT, the computerized version of Psychological Abstracts, reveals that in 1992 there was only 1 listing including the keyword "T-maze," with 5 in 1987, 25 in 1982, and 25 in 1977.

The $\phi$-maze was developed in my laboratory to solve the problem of automating T-maze-type studies. The $\phi$-maze is shaped like a T-maze in which the left and right arms each lead back to the base of the T via two $90^{\circ}$ turns. The rat is required to return to the starting point to receive the reinforcer and to end the trial, thus positioning itself for the start of the next trial. The doors, feeder, and discriminative stimuli are under computer control, and the position of the rat within the $\phi$-maze is monitored by the computer via infrared light-emitting diodes (LEDs) and photodarlingtons mounted throughout the maze. Data collection and stimulus control are thus fully automated, requiring no experimenter involvement during the course of the session.

The $\phi$-maze differs from other automated T-mazes in two important ways. First, the reinforcer is delivered in the

The $\phi$-maze was constructed with the assistance of a GOAP grant from the School of Arts and Sciences, Indiana University-Purdue University Fort Wayne. I thank J. A. Cook and three anonymous reviewers for their helpful criticisms of a preliminary version of this manuscript. Correspondence should be addressed to W. J. Wilson, Department of Psychological Sciences, Indiana University-Purdue University Fort Wayne, 2101 Coliseum Blvd. East, Fort Wayne, IN 46805 (e-mail: wilsonj@smtplink.ipfw.indiana.edu). startbox, thus ensuring that the subject's return to the startbox is motivated and reinforced by the delivery of the reinforcer for the current trial. In similar mazes, the reinforcer has been delivered in either the end of the $T$ or in the return arms. In this case, the subject's return to the startbox is motivated by the possibility of reward on the next trial, and thus is part of the next trial, although usually not discussed as such. Second, the $\phi$-maze is fully automated, unlike other automated T-mazes, discussed below, which in fact require the experimenter to control doors or deliver reinforcers. The $\phi$-maze is the first T-maze to incorporate both of these features.

The $\phi$-maze is based loosely on the behavioral apparatus described by Berger (1977), in that the discrimination response requires locomotion down one of two alleyways, and the rat returns to the startbox to receive a reinforcer. However, Berger's apparatus required the rat to make a leverpress or nosepoke response at the end of the alleyway before returning to the startbox where the reinforcer was delivered, and the rat returned by retracing its steps through the same alley that led to the manipulandum. Other experimenters have used mazes topologically more similar to the $\phi$-maze and requiring only locomotor responses. Lashley (1912) described the Yerkes' discrimination box, an apparatus like that of Berger except that the rat returned to the startbox via separate alleyways, and reinforcers were delivered in these alleys, not in the startbox. Hunter (1920) used his "temporal maze" to examine the acquisition of complex patterned responses in rats. This maze was identical in shape to the $\phi$-maze, but lacked doors and specific startboxes and goalboxes. The rat was required to follow a particular route through the maze, sometimes retracing its steps, in order to be reinforced.

Holman (1966) conducted T-maze studies with rats in a maze that included alleys leading from the end of each of the two arms, where reinforcers were delivered, to the startbox. Levine, Hull, Buchwald, and Villablanca (1978) have described discrimination experiments in cats 
using a maze similar to that of Holman. Johnson (1913) studied auditory discrimination in dogs in what was essentially a $\phi$-maze, except that reinforcers were delivered in the return arms just outside the startbox. The mazes of Berger (1977) and Holman were automated; the mazes used by Lashley (1912), Hunter (1920), Levine et al., and Johnson required constant experimenter involvement.

Nakagawa (1993) has recently published an experiment in which a commercially available automated T-maze was employed. This automated T-maze, however, appears topologically more similar to a two-lever operant chamber than a T-maze, because the visual discriminative stimuli and the levers are located on the wall opposite the startbox. The apparatus appears to have great potential for visual discrimination studies; however, it requires the rat to press a lever to indicate its choice. This necessitates extensive shaping: Nakagawa indicated that 15 days of leverpress training were required before discrimination training could begin. It is likely that the location of the manipulanda distant from the feeder contributed to the need for extensive training; we have also found that shaping rats to press levers located at some distance from the feeder requires two shaping sessions under the best of circumstances, and sometimes more (Wilson \& Cook, 1989). In the $\phi$-maze, the only response required by the rat is locomotion, and shaping of the rat's behavior is not necessary.

\section{THE $\phi$-MAZE}

\section{Plan of the $\phi$-Maze}

The $\phi$-maze consists of a start/goalbox ( $\mathrm{s} / \mathrm{g}$ box) from which a central alley leads to a choice point. From the choice point, left and right arms return to the s/g box. Figure 1 presents a plan of the maze. The maze is constructed of 0.25 -in. $(0.6 \mathrm{~cm})$ acrylic plastic: the walls are black, the floor is translucent white, and the top is clear. Automated doors of translucent white acrylic separate the $\mathrm{s} / \mathrm{g}$ box from the central alley and the two return arms. An automated mechanism in the $\mathrm{s} / \mathrm{g}$ box can deliver appetitive reinforcers. Five infrared LED-photodarlington pairs located throughout the maze can record the rat's position. Banks of lights located beneath the floor of the central alley and the left and right arms can serve as visual cues. If desired, speakers could be mounted at the choice point or in the left and right arms to provide auditory cues. The total cost of materials for the $\phi$-maze, including the parallel port interface for the computer, was less than $\$ 700$ in the summer of 1991 .

\section{Alleyways}

A cross section of an alleyway is presented in Figure 2. Each alley is $12.2 \mathrm{~cm}$ wide and $19.3 \mathrm{~cm}$ from floor to ceiling. Where they occur, the infrared LEDs and photodarlingtons are located $3.8 \mathrm{~cm}$ from the floor, on opposite sides of the alley. Beneath the floor of the central alley and the two return arms are $28-\mathrm{V}$ lamps, located as shown in Figures 1 and 2.

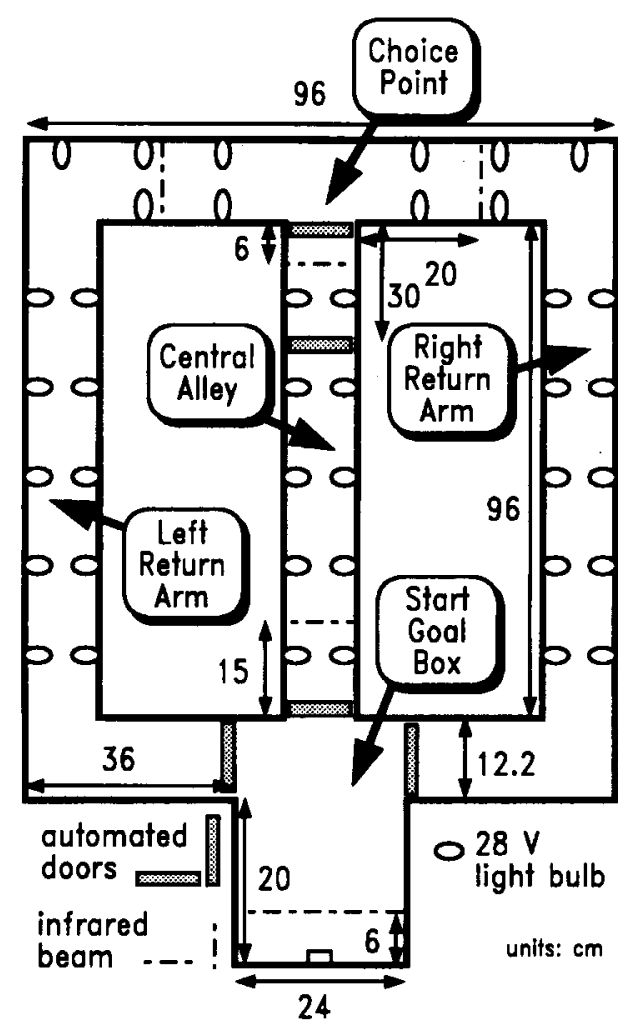

Figure 1. Plan of the $\phi$-maze. Walls are black acrylic; floor is translucent white; and ceiling is clear.

\section{Doors}

The five guillotine doors are constructed of translucent white acrylic and slide within aluminum channels. The top of each door is attached by a short length of chain to a metal lever. The opposite end of the lever is counterbalanced with a lead weight sufficiently heavy to allow the door to close slowly by the force of gravity. A continuous-duty solenoid (Guardian Electric 4-C-24V) attached to the lever can open the door and hold it open indefinitely. Each door is normally programmed to close when the rat is not near it, but because it is closed by gravity and is carefully counterbalanced, it would not harm the rat if the rat happened to be under it when it closed. The doors open to a height of $6.5 \mathrm{~cm}$ in approximately $0.4 \mathrm{sec}$; they close in approximately $0.6 \mathrm{sec}$.

\section{Feeder}

Quartered Froot Loops (Kellogg's) are used as reinforcers. A Lehigh Valley Equipment pellet dispenser has been modified to deliver them automatically. The aluminum plate of the pellet dispenser has been replaced with an acrylic disk ( $19 \mathrm{~cm}$ in diameter) containing twelve $2-\mathrm{cm}$ holes equally spaced around its perimeter. A $1.5-\mathrm{cm}$ deep bottomless plastic cup is cemented to each hole. Brief operation of the feeder results in a $30^{\circ}$ rotation of the disk, which aligns one of the holes with an opening that allows the contents of the cup to drop into the feeder trough in the $\mathrm{s} / \mathrm{g}$ box. Because of the increased momentum 


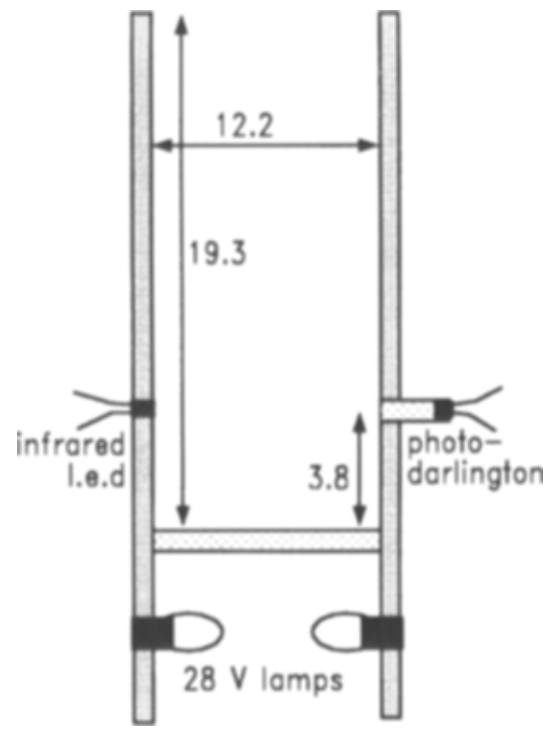

Figure 2. Cross section of an alley of the $\phi$-maze illustrating the positions of the light-emitting diode (LED), photodarlington, and lamps. All measurements are in centimeters.

inherent in the acrylic disk compared with the original aluminum plate, the timing of the operating pulse is critical. A pulse of duration less than about $10 \mathrm{msec}$ causes the disk to spin freely, dropping the contents of several cups; a pulse of approximately $14 \mathrm{msec}$ turns the disk $30^{\circ}$ and then locks it in place.

\section{Electronics}

An IBM-PC-compatible computer controls the maze and records data about the rat's performance via a locally manufactured parallel port interface, based on a design presented by Mangieri (1991). Morris (1992), Lavond and Steinmetz (1989), and Arnold and Hastings (1984), to name a few, have presented designs for other similar interfaces. Optoisolation was incorporated in the design to protect the computer from unexpected voltage surges in the equipment. The interface allows the control of as many as 12 stimuli and the recording of five digital inputs, in this case the five photodarlingtons. The interface is based on TTL logic and is powered by a $12-\mathrm{V}$ power supply internally stepped down to $5 \mathrm{~V}$. Stimuli are controlled by power transistors, each of which is capable of driving as much as $1.5 \mathrm{~A}$.

The five infrared LED-photodarlington pairs located as shown in Figures 1 and 2 are electronically quite simple. Each LED (Honeywell SEP8705-003) is powered by the same $12-\mathrm{V}$ source that drives the interface and is connected in series with a $220-\Omega$ resistor. The LEDs are illuminated whenever the interface is powered. Each photodarlington (Honeywell SD5410-002) is mounted inside of a 0.6-cm-inside-diameter, $2.0-\mathrm{cm}$-long black tube, and is aimed directly at the LED opposite it. Each photodarlington will allow $5 \mathrm{~V}$ to flow to one of the five digital inputs of the interface when light from the LED falls on it. If the light from the LED is blocked, the photo- darlington stops conducting, and the input to the interface goes to ground.

\section{Software}

The $\phi$-maze is controlled by an IBM-PC-compatible 8088-based computer running at $12 \mathrm{mHz}$. In 8088-based computers, the ROM BIOS clock is accurate only to the nearest $0.055 \mathrm{sec}$, approximately (see Beringer, 1992, for a discussion of various means of overcoming this limitation of the ROM BIOS timer). I achieve accurate timing by means of a different approach; I program the internal timer chip (8253-5, independent of the BIOS timer, and usually used for sound generation) to activate a flip-flop every $0.01 \mathrm{sec}$ (see Norton, 1985, pp. 148-150). When the flip-flop changes state, the software executes a loop of code that, in turn, increments a timing counter, examines the input lines, records any responses, and updates any output lines. If a response has occurred, as determined by a change in the status of one of the sensors, the latency of the response is saved to an array in memory. Because this approach utilizes software-dependent timing, and because both screen- and disk-access via the BIOS are relatively slow and would disrupt that timing, nothing is written to screen or disk until the trial ends.

My experimental control software is written in Turbo Pascal 3.0 (Borland International, 1985) and requires the user to be knowledgeable in Pascal. More user-friendly software that could be used to control the $\phi$-maze has been described by Schneider (1988) and Walter and Palya (1984, Palya \& Walter, 1993).

\section{USE OF THE $\phi$-MAZE}

A trial in the $\phi$-maze begins when the door to the central alley is opened. This door closes behind the rat as it leaves the $\mathrm{s} / \mathrm{g}$ box. At some time while the rat is traversing the maze, both doors between the return arms and the $\mathrm{s} / \mathrm{g}$ box are opened. I typically open the doors once the rat has selected either the left or right arm; the trial ends when the rat returns to the feeder. If the correct arm has been chosen, a reinforcer is then delivered. It is also possible to open only one door, thus causing the rat to reach a dead end if the incorrect arm is selected. In this case, the rat would be required to retrace its steps to the choice point and select the correct arm. In studies requiring the holding area, the door behind the rat closes as the rat reaches the end of the central alley, and the door to the choice point opens after an appropriate delay.

Rats are habituated to the $\phi$-maze by being reinforced for leaving the s/g box via the central alley and returning through either the left or right arms. I routinely run habituation sessions until the rat has completed a certain number of trials in a predetermined amount of time over 2 successive days. For example, in 6 rats required to run 40 trials in less than 40 min, the median number of sessions required to reach this criterion was 3.5 (including the criterion sessions). In another group of 6 rats required to complete 40 trials in less than $30 \mathrm{~min}$, the median number of sessions was 6.5 . It should be noted that 
in rats that are familiar with the maze and the task, a typical trial might require as little as $10 \mathrm{sec}$.

The $\phi$-maze is ideally suited to studies of choice behavior. In my laboratory it has been used successfully in studies of visual and spatial alternation (Wilson, Peters, Felton, \& Galloway, 1994; Wilson \& Steinbronn, 1993; Wilson et al., 1992), visual discrimination (Wilson et al., 1992), and (delayed) nonmatching-to-sample (unpublished data from my laboratory).

Discrimination sessions can be conducted for a fixed number of sessions or until some criterion has been achieved. For example, in a study of spatial alternation (Wilson et al., 1994) we continued the sessions until the rats had received 40 reinforcers in 50 or fewer trials in two successive sessions. The median number of sessions required to reach this criterion was 6 (minimum 2, maximum 9 , including criterion sessions) for a group of 7 rats.

As in all discrimination experiments, the accuracy of the choice response can be monitored. In addition to this, it is possible to monitor several types of errors made by the rats. For example, in an alternation task, the rat might repeatedly select the incorrect arm, failing to alternate. This type of perseverative error does not represent a memory deficit, because perseveration, like correct responding, requires knowledge of the previous response.

Within a trial, it is possible for the rat to make several types of errors. The rat might reenter the central arm or return to the exit door of the $\mathrm{s} / \mathrm{g}$ box; both of these responses interfere with the successful completion of the trial, thereby delaying reinforcement. The rat might also enter one arm of the maze, then backtrack and enter the other, sometimes repeatedly within the same trial. I have observed all of these types of errors.

Finally, it is possible to monitor various latencies within each trial. I have found it useful to examine how long it takes the rat to complete each of the following tasks: start a trial, run down the central alley, select an arm of the maze, and return to the feeder. These latencies are affected differently by drug manipulations, suggesting that they might reflect independent underlying processes. For example, the latencies to initiate a trial and to run through the central alley are increased in a dose-dependent manner by both the centrally and peripherally acting forms of scopolamine, but only central blockade increases the latency to return to the startbox for the reinforcer (Wilson \& Steinbronn, 1993).

\section{DISCUSSION}

The $\phi$-maze is a novel implementation of an apparatus once widely used in behavioral research, the T-maze. Locomotor responses alone on the part of the rat are used to make discrimination responses. The fully automated nature of the apparatus precludes the need to handle the rat during the session, as is necessary in T-mazes. The automation of the maze is made possible in part by the delivery of the reinforcer in the startbox, which motivates the rat to return to the startbox to conclude a trial.
Because the $\phi$-maze is inexpensive to construct and easily automated, it should prove to be a valuable tool for learning and memory studies in the rat. Alternation, visual discrimination, and delayed matching-to-sample experiments can all be conducted with ease in the apparatus. The $\phi$-maze can also serve as a circular runway if only the perimeter pathway is used.

Because there is no need to handle the rat, and because stimulus control and data recording are carried out by the computer, the session is no more difficult to conduct than is a session using an operant chamber. The various latency measures available, and the different types of within-trial errors possible, provide behavioral measurements that are not readily available in an operant chamber.

Its versatility, low cost, and ease of use make the $\phi$-maze a potentially valuable tool in studies of learning and memory. I continue to explore the uses of the $\phi$-maze in studies of the neurochemical and neuroanatomical substrates of working memory, and would welcome any interaction with others interested in the use of this apparatus.

\section{REFERENCES}

ARnold, N. D., \& Hastings, L. (1984). A bit-addressable I/O interface for microcomputers. Behavior Research Methods, Instruments, \& Computers, 16, 135-136.

BERGER, B. D. (1977). An animal model for the measurement and manipulation of problem solving ability. In E. S. Gershon, R. H. Belmaker, S. S. Kety, \& M. Rosenbaum (Eds.), The impact of biology on modern psychiatry (pp. 187-194). New York: Plenum.

BERRINGER, J. (1992). Notes on Graves and Bradley's timer algorithm. Behavior Research Methods, Instruments, \& Computers, 24, 453-455.

Borland InTERnational (1985). Turbo Pascal (Version 3.0) [Computer software]. Scotts Valley, CA: Author.

Holman, E. W. (1966). Tests for spontaneous alternation. Psychological Review, 73, 427-436.

HUNTER, W. S. (1920). The temporal maze and kinesthetic sensory processes in the white rat. Psychobiology, 2, 1-17.

Johnson, H. M. (1913). Audition and habit formation in the dog. Behavior Monographs, 2, 1-78.

LASHLEY, K. S. (1912). Visual discrimination of size and form in the white rat. Journal of Animal Behavior, 2, 310-331.

Lavond, D. G., \& SteinmetZ, J. E. (1989). An inexpensive interface for the IBM PC/XT and compatibles. Behavior Research Methods, Instruments, \& Computers, 21, 435-440.

Levine, M. S., Hull, C. D., Buchwald, N. A., \& Villablanca, J. R. (1978). Effects of caudate nuclei or frontal cortical ablations in kittens: Motor activity and visual discrimination performance in neonatal and juvenile kittens. Experimental Neurology, 62, 555-569.

MANGIERI, A. M. (1991, April). Build a parallel printer port I/O interface. ComputerCraft, pp. 54-58, 76-79.

MoRRIS, C. C. (1992). Using the IBM-compatible microcomputer's serial port as an input-output interface. Behavior Research Methods, Instruments, \& Computers, 24, 456-460.

NAKAGAWA, E. (1993). Matching and nonmatching concept learning in rats. Psychobiology, 21, 142-150.

NoRTON, P. (1985). The Peter Norton programmer's guide to the IBM PC. Bellevue, WA: Microsoft Press.

Palya, W. L., \& Walter, D. E. (1993). A powerful, inexpensive experiment controller or IBM PC interface and experiment control language. Behavior Research Methods, Instruments, \& Computers, 25, $127-136$

SCHNEIDER, W. (1988). Micro Experimental Laboratory: An integrated system for IBM PC compatibles. Behavior Research Methods, Instruments, \& Computers, 20, 206-217.

Walter, D. E., \& Palya, W. L. (1984). An inexpensive experiment controller for stand-alone applications or distributed processing net- 
works. Behavior Research Methods, Instruments, \& Computers, 16, 125-134.

WILsON, W. J., \& CooK, J. A. (1989). Acquisition and reversal of visual discrimination in rats: Effects of cholinergic manipulations [Abstract]. Bulletin of the Psychonomic Society, 27, 502.

Wilson, W. J., Peters, D. W., Felton, M. L., \& Galloway, P. H. (1994). Delayed spatial alternation performance of rats in a phi-maze: Scopolamine effects. Society for Neuroscience Abstracts, $20,149$. Wilson, W. J., \& Steinbronn, N. C. (1993). Dose-dependent disrup- tion by scopolamine of spatial working memory performance of rats in a phi $(\phi)$-maze. Society for Neurascience Abstracts, 19, 1811.

Wilson, W. J., Steinbronn, N. C., Lopshire, J. C., Galloway, P. H., Kellenberger, S. A., Hartman, A. M., \& Bennett, R. L. (1992), Differences in spatial and visual working memory performance of rats in a phi $(\phi)$-maze. Society for Neuroscience Abstracts, 18, 1423.

(Manuscript received August 8, 1994; revision accepted for publication May 26, 1995.) 